\title{
Probability Map Building of Uncertain Dynamic Environments with Indistinguishable Obstacles*
}

\author{
Myungsoo Jun ${ }^{\dagger}$ and Raffaello D'Andrea \\ Sibley School of Mechanical and Aerospace Engineering \\ Cornell University \\ Ithaca, NY 14853-7501, USA
}

Key Words: Autonomous vehicle, probability map building, uncertain dynamic environments, data association

\begin{abstract}
This paper proposes a method to build a probability map of the environment for navigation. It is assumed that the environment has multiple indistinguishable moving obstacles and the vehicle has limited sensor range and, therefore, lacks global information. The probability map is updated through the measurement and the probabilistic model of the obstacles. The model is obtained from a priori statistics of their movement. Probabilistic data association method is used to track multiple obstacles even after the vehicle loses tracking of some obstacles. The error bound of the algorithm is also analyzed.
\end{abstract}

\section{Introduction}

Unmanned aerial vehicles (UAV's) are being used more and more in a number of civilian and military applications, e.g., remote monitoring of traffic, search and rescue operations, surveillance, etc. This has intrigued the control community and research on the control and design of UAV's is being actively conducted. One of main issues in the design of autonomous vehicles is putting autonomy and intelligence to the vehicles. This includes autonomous navigation to the destination. For such navigation, the vehicles should be able to properly represent the environment and to plan a path to the destination.

The problems of building a map of the environment and finding a collision-free path have been an active research area in the robotics and artificial intelligence communities. The robot path planning methods can be categorized into three approaches: cell decomposition methods, roadmap methods and artificial potential field methods [6]. It can be noticed from the classifi-

* Research sponsored by AFOSR Grant F49620-01-1-0361

†Corresponding author; email mj73@cornell.edu; cation that the methods for path planning are closely related to the representation of the environment. Cell decomposition methods rely on the discretization of the configuration space. Roadmap methods represent the environment by a network of graphs such as visibility graph and Voronoi graph. Artificial potential field methods use a potential function for the representation of the environment, which is defined to provide attractive forces toward the destination and repulsive forces from obstacles.

When there exists uncertainty in the information, a probabilistic approach is commonly used. The most widespread method for building a probability map of an uncertain environment is grid-based occupancy maps (See $[3,7,10]$ and references therein). Occupancy values for each grid cell are determined based on sensor readings and by applying the conditional probability of occupancy using Bayes' rule. These values are determined by the sensor characteristics, the location of the sensors and the measurement methods. The grid-based occupancy map approach, however, is mostly applied to static environments. Thrun [9] adopted a simple exponential decay factor which puts more weight on recent sensor readings in order to cope with dynamic environments. As the author acknowledges, this approach does not fully model dynamic environments but just adapts to changes. Cox et al. [1] used a Bayesian multiple hypothesis framework to build and maintain a world model of a dynamic environment. Their approach, however, focused on modeling dynamic environments by simple geometric primitives and extracting features from multiple hypothesis and sensor readings. Jun et al. [5] proposed a method to build a probability map of uncertain dynamic environments by using a probabilistic obstacle model. A probability distribution function was used for state transition of obstacles, which can be obtained from a priori information on the obstacles. The results, however, did not specify a data association problem. 
The information on the location of previously detected obstacles is important for planning a return path if the mission requires the vehicle to return after it reaches the target. Furthermore, it is necessary to interpret measured data correctly so that the constructed map can represent the environment properly. If the obstacles are distinguishable from each other, association can be determined uniquely by extracting features from measurement (See $[1,8,4])$. However, most research of the data association problems concerned distinguishable target tracking and did not consider the case when the vehicle loses objects from its sensor range.

This paper presents how to update the probability map of an environment when the vehicle loses tracking of objects and proposes a method to associate measured data with indistinguishable obstacles by using probabilistic approach.

The paper is organized as follows: Section 2 briefly explains the notation used in the paper. Section 3 presents a probability map building algorithm in uncertain dynamic environment with multiple indistinguishable obstacles. Section 4 describes how to associate the measured data with indistinguishable obstacles and error bound of it. Section 5 describes the simulation platform and Section 5.2 discusses simulation results.

\section{Definitions and Notation}

Given that there are $l$ moving obstacles. We assume that obstacles are indistinguishable from each other. Let $\mathcal{R}$ be the region of consideration with 2-dimension and $\mathcal{X}=\mathcal{R} \times \Theta$ where $\times$ means Cartesian product and $0 \leq \theta \leq 2 \pi, \theta \in \Theta$ be the state-space of obstacles. Let $\mathcal{D}$ be the index set of detected obstacles until current time. Let $\mathcal{D}^{c}$ denote the complement of the set $\mathcal{D}$. We denote sampling time by $\Delta t$, the current time by $T \Delta t$, measured data at $k$-th sampling time by $m_{k}$, and sequence of measured data up to time $T \Delta t$ by $m^{(T)}$, viz., $m^{(T)}=\left\{m_{1}, m_{2}, \cdots, m_{T}\right\}$. We define $\mathbf{x}_{T}^{i} \in \mathcal{X}$ to be the state of the obstacle $i$ at time $T \Delta t$. All probability density functions (pdf) are represented by $f(\cdot)$.

\section{Modeling of Dynamic Environments}

This section describes a method to build a probability map of the environment with multiple dynamic obstacles. It is assumed that there are no static obstacles in the environment, as is common for unmanned aerial vehicles. It is also assumed that the vehicle has limited sensor range.

It is assumed that the initial distribution of each obstacle, $f\left(\mathbf{x}_{0}^{i} \mid m^{(0)}\right), 1 \leq i \leq i$, is uniform because the vehicle does not have any information on the location of obstacles before it starts to move. The vehicle gathers information from sensor readings as it moves. Due to the limited sensor range, the vehicle may not detect all obstacles at each measurement. Whenever the vehicle senses an obstacle, uncertainty on the location of the obstacle greatly decreases. The uncertainty is not zero due to sensor noise. The vehicle modifies the distribution function of the detected obstacle based on this location information. If the vehicle no longer detects an obstacle, uncertainty of the obstacle's location, which was sensed at a previous time, increases since obstacles are moving. In such a case, the vehicle should calculate the distribution function of the obstacle based on a priori statistics on obstacle movement and previous information on the location of the obstacle from sensor readings. The probability map building algorithm in uncertain dynamic environments can be formulated by using Bayesian estimation [5].

The recursive form of the pdf of the staten of each obstacle given measured data $m^{(T)}$ can be obtained as follows:

$$
\begin{aligned}
& f\left(\mathbf{x}_{T}^{i} \mid m^{(T)}\right) \\
= & f\left(\mathbf{x}_{T}^{i} \mid m_{1}, m_{2}, \cdots, m_{T}\right) \\
= & \frac{f\left(m_{T} \mid m_{1}, \cdots, m_{T-1}, \mathbf{x}_{T}^{i}\right) f\left(\mathbf{x}_{T}^{i} \mid m_{1}, \cdots, m_{T-1}\right)}{f\left(m_{T} \mid m_{1}, \cdots, m_{T-1}\right)} \\
= & \frac{f\left(m_{T} \mid \mathbf{x}_{T}^{i}\right) f\left(\mathbf{x}_{T}^{i} \mid m_{1}, \cdots, m_{T-1}\right)}{f\left(m_{T} \mid m_{1}, \cdots, m_{T-1}\right)} \\
= & \frac{f\left(m_{T} \mid \mathbf{x}_{T}^{i}\right) f\left(\mathbf{x}_{T}^{i} \mid m^{(T-1)}\right)}{f\left(m_{T} \mid m^{(T-1)}\right)} \\
= & \frac{f\left(m_{T} \mid \mathbf{x}_{T}^{i}\right) f\left(\mathbf{x}_{T}^{i} \mid m^{(T-1)}\right)}{\int_{\mathcal{X}} f\left(m_{T} \mid \mathbf{x}_{T}^{i}\right) f\left(\mathbf{x}_{T}^{i} \mid m^{(T-1)}\right) d \mathbf{x}_{T}^{i}}
\end{aligned}
$$

Here, $f\left(m_{T} \mid \mathbf{x}_{T}^{i}\right)$ is usually called measurement model and denotes the pdf that describes how sensor measurements $m_{T}$ are generated when the obstacle $i$ has the state $\mathbf{x}_{T}^{i}$ at time $T \Delta t$, viz., the obstacle $i$ is at position $(x, y) \in \mathcal{R}$ with the direction $\theta \in \Theta$ at time $T \Delta t$.

The term $f\left(\mathbf{x}_{T}^{i} \mid m^{(T-1)}\right)$ is called the prior pdf of the state because it is the pdf of the obstacle $i$ 's state at time $T \Delta t$ given the measurement up to time $(T-1) \Delta t$, thus, it can be interpreted as a prediction step. It is obtained from the Chapman-Kolmogorov Equation and is given by

$f\left(\mathbf{x}_{T}^{i} \mid m^{(T-1)}\right)=\int_{\mathcal{X}} f\left(\mathbf{x}_{T}^{i} \mid \mathbf{x}_{T-1}^{i}\right) f\left(\mathbf{x}_{T-1} \mid m^{(T-1)}\right) d \mathbf{x}_{T-1}^{i}$.

The term $f\left(\mathbf{x}_{T}^{i} \mid \mathbf{x}_{T-1}^{i}\right)$ is usually called system model or motion model and is related to state transition with time and is the probability that the obstacle $i$ will have the state $\mathbf{x}_{T}^{i} \in \mathcal{X}$ at time $T \Delta t$ when the obstacle $i$ has the state $\mathbf{x}_{T-1}^{i} \in \mathcal{X}$ at time $(T-1) \Delta t$. This can be calculated if information is available on the velocity of 


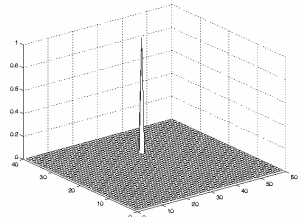

(a)

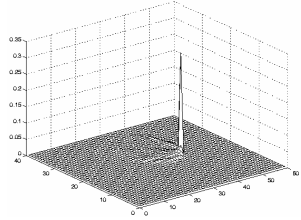

(c)

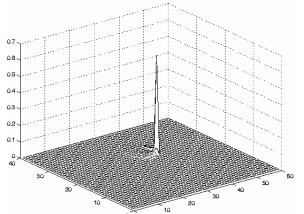

(b)

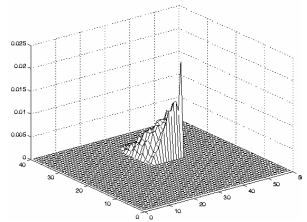

(d)
Figure 1: Probability map (a) when the vehicle detects an obstacle. (Measurement update), and (b) when the vehicle loses tracking of the obstacle after 0.5 second time propagation, (c) 1 second time propagation and (d) 1.5 second time propagation.

obstacles, statistics on changes of direction, and so on. This prediction is corrected by the new measurement $m_{T}$ by using the Eq. (1).

Based on the Eq. (1) and Eq. (2), a probability map of the environment at time $T \Delta t$ can be defined as follows*

$$
\operatorname{Map}_{T}(x, y) \triangleq 1-\prod_{i=1}^{l}\left(1-\int_{\Theta} f\left(x, y, \theta \mid m^{(T)}\right) d \theta\right)
$$

if $f\left(\mathbf{x}_{T}^{i} \mid m^{(T)}\right)=f\left(x, y, \theta \mid m^{(T)}\right), \mathbf{x}_{T}^{i}=(x, y, \theta) \in \mathcal{X}$. Notice that $\operatorname{Map}_{T}(x, y)$ is not a pdf with respect to $x$ and $y$.

The Figure 1 shows an example of a probability map. It can be noticed that the uncertainty in information on the locations of obstacles increases once they are out of the vehicle's sensor range

\section{Obstacle Association}

It is not always required for the vehicle to calculate the estimated the locations of obstacles after it loses tracking of them. If the mission of the vehicle ends after it reaches the target and obstacles are slower than the vehicle, the vehicle does not have to estimate the track of obstacles after it passes around them and leaves them behind because it is less probable for the vehicle to re-enter the area around where it had detected them. However, the information on the location of previously detected obstacles is important in planning a return

\footnotetext{
*The product operation is performed for the pdf $f(\cdot)$ of each obstacle even though the same symbol $f(\cdot)$ is used to denote each pdf.
}

path if the mission requires the vehicle to return to its origin after it reaches the target.

When the vehicle detects an obstacle on its return to the origin, it should determine whether the currently detected one is a new one or previously detected one. If the currently detected one is presumed to be previously detected one, the vehicle also should estimate which obstacle it is among previously detected ones in order to properly update the corresponding pdf. If the obstacles are distinguishable, association can be determined uniquely by extracting features from measurement and comparing hypothesis. When obstacles are indistinguishable, another data association is needed. The method used in the paper is to associate measured data with obstacles according to the probability distribution function.

\subsection{Obstacle Association Algorithm}

Let us assume that the vehicle detects an obstacle at time $T \Delta t$. We define $\mathbf{x}_{T} \in \mathcal{X}$ to be the state of the obstacle at time $T \Delta t$. We assume that $f\left(\mathbf{x}_{T} \mid m^{(T-1)}\right)$ has uniform distribution. Our objective is how to associate $f\left(\mathbf{x}_{T} \mid m^{(T)}\right)$ with $f\left(\mathbf{x}_{T}^{i} \mid m^{(T)}\right), 1 \leq i \leq l$.

From Bayes' rule, $f\left(\mathbf{x}_{T} \mid m^{(T)}\right)$ can be expressed as

$$
\begin{aligned}
f\left(\mathbf{x}_{T} \mid\right. & \left.m^{(T)}\right) \\
& =\frac{f\left(m_{T} \mid \mathbf{x}_{T}\right) f\left(\mathbf{x}_{T} \mid m^{(T-1)}\right)}{\int_{\mathcal{X}} f\left(m_{T} \mid \mathbf{x}_{T}\right) f\left(\mathbf{x}_{T} \mid m^{(T-1)}\right) d \mathbf{x}_{T-1}} .
\end{aligned}
$$

Let $g(x, y)$ and $h_{i}(x, y)$ to be the functions on $\mathcal{R}$ defined by

$$
\begin{aligned}
g(x, y) & \triangleq \int_{\Theta} f\left(\mathbf{x}_{T} \mid m^{(T)}\right) d \mathbf{x}_{T} \\
& \triangleq \int_{\Theta} f\left(x, y, \theta \mid m^{(T)}\right) d \theta \\
h_{i}(x, y) & \triangleq \int_{\Theta} f\left(\mathbf{x}_{T}^{i} \mid m^{(T-1)}\right) d \mathbf{x}_{T}^{i} \\
& \triangleq \int_{\Theta} f\left(x, y, \theta \mid m^{(T-1)}\right) d \theta,
\end{aligned}
$$

respectively ${ }^{\dagger}$. And let us define $p_{i}$ to be

$$
p_{i} \triangleq \int_{\mathcal{R}} g(x, y) \cdot h_{i}(x, y) d x d y, \quad 1 \leq i \leq l .
$$

Then, the quantity $p_{i}$ is proportional to the probability that the detected obstacle is obstacle $i$. When $i \in \mathcal{D}^{c}$, we have $p_{i}=\frac{1}{2 \pi R}$ where $R=\int_{\mathcal{R}} d x d y$ since $f\left(\mathbf{x}_{T}^{i} \mid m^{(T-1)}\right)$ is uniform for $i \in \mathcal{D}^{c}$. This value will be used as a threshold to determine whether the detected obstacle is a new one or previously detected one.

\footnotetext{
†Note that the pdf $f\left(x, y, \theta \mid m^{(T)}\right)$ in the Eq. (5) and the pdf $f\left(x, y, \theta \mid m^{(T-1)}\right)$ in the Eq. (6) have different distribution although they are expressed by same symbol $f(\cdot)$. The pdf $f(\cdot)$ in the Eq. (5) is the posterior pdf of $\mathbf{x}_{T}$ and the pdf $f(\cdot)$ in the Eq. (6) is the prior pdf of $\mathbf{x}_{T}^{i}$.
} 
Define $k \triangleq \arg \max _{i \in \mathcal{D}} p_{i}$. First, let us consider the case when $p_{k}>\frac{1}{2 \pi R}$. In this case, the currently detected obstacle will be considered to be one of the previously detected obstacles and will be associated with the obstacle $k$, which has maximum value of $p_{i}$. Since the currently detected obstacle is considered to be the obstacle $k$, the pdf $f\left(\mathbf{x}_{T}^{k} \mid m^{(T)}\right)$ should be updated in accordance with the measured data $m^{(T)}$ by Eq. (1).

Next, consider the case when $p_{k}<\frac{1}{2 \pi R}$. In such case, the currently detected obstacle will not be considered to be the one of previously detected obstacles and will be associated with arbitrary obstacle $j$ which is in the set $\mathcal{D}^{c}$. After association, modify the set $\mathcal{D}$ and update the pdf $f\left(\mathbf{x}_{T}^{j} \mid m^{(T)}\right)$ accordingly. The association algorithm is summarized in Algorithm 1.

1. Set $f\left(\mathbf{x}_{T} \mid m^{(T-1)}\right)=\frac{1}{2 \pi R}$;

2. Get measurement $m_{T}$ and calculate $f\left(\mathbf{x}_{T} \mid m^{(T)}\right)$ by the Eq. (4);

3. Calculate the functions $g(x, y)$ and $h_{i}(x, y)$ by the Eq. (5) and Eq. (6);

4. Calculate $p_{i}$ in the Eq. (7) and find $k=\arg \max _{i \in \mathcal{D}} p_{i}$

5. If $p_{k}>\frac{1}{2 \pi R}$, associate the currently detected obstacle with the obstacle $k$ and update the pdf $f\left(\mathbf{x}_{T}^{k} \mid m^{(T)}\right)$; otherwise, associate the currently detected obstacle with the obstacle $j \in \mathcal{D}^{c}$ and update the pdf $f\left(\mathbf{x}_{T}^{j} \mid m^{(T)}\right)$;

6. Update the set $\mathcal{D}$ accordingly.

Algorithm 1: Algorithm for Obstacle Association

\subsection{Obstacle Association Error}

The section analyzes the probability that the currently detected obstacle will be associated with a wrong obstacle. We assume that $m \neq 0 \leq l$ obstacles were previously detected, that is, the number of elements of the set $\mathcal{D}$ is $m$. If we define $\mathbf{i}$ to be a random variable denoting the index of the currently detected obstacle, $P\left(\mathbf{i}=i \mid m^{(T)}\right)$ denotes the probability that the currently detected obstacle is the obstacle $i$ given the measurement $m^{(T)}$ and it can be said that

$$
P\left(\mathbf{i}=i \mid m^{(T)}\right)=\frac{p_{i}}{\sum_{j=1}^{l} p_{j}} .
$$

Then, the probability that the currently detected obstacle is not the obstacle $i$ given the measurement $m^{(T)}$ is

$$
P\left(\mathbf{i} \neq i \mid m^{(T)}\right)=1-P\left(\mathbf{i}=i \mid m^{(T)}\right)=1-\frac{p_{i}}{\sum_{j=1}^{l} p_{j}} .
$$

This is the probability that the currently detected obstacle will be associated with a wrong obstacle when the currently detected obstacle is associate with the obstacle $i$.

Let $P($ error $)$ be the probability that the currently detected obstacle will be associated with a wrong obstacle. The bound of $P($ error $)$ when $p_{k}>\frac{1}{2 \pi R}$ can be expressed as in the next proposition.

Proposition 1 If $p_{k}>\frac{1}{2 \pi R}$, then

$$
\frac{l-1}{2 \pi R \cdot p_{k}+l-1} \leq P(\text { error }) \leq \frac{l-1}{l}
$$

where $k=\arg \max _{i \in \mathcal{D}} p_{i}$.

Proof: Let us assume that $m$ obstacles were previously detected.

Since $f\left(\mathbf{x}_{T}^{i} \mid m^{(T-1)}\right)=\frac{1}{2 \pi R}$ if $i \in \mathcal{D}^{c}$ and the currently detected obstacle is associated with the obstacle $k$ if $p_{k}>\frac{1}{2 \pi R}$, it can be said that $p_{i}=\frac{1}{2 \pi R}$ for $i \in \mathcal{D}^{c}$. Therefore, we have

$$
P(\text { error })=1-\frac{2 \pi R \cdot p_{k}}{2 \pi R \sum_{i \in \mathcal{D}} p_{i}+(l-m)} .
$$

The maximum occurs when $p_{i}=p_{k}$ for all $i \in \mathcal{D}$ and $m=l$, thus,

$$
P(\text { error }) \leq 1-\frac{2 \pi R \cdot p_{k}}{2 \pi R \cdot l \cdot p_{k}}=\frac{l-1}{l} .
$$

The minimum occurs when $m=1$, thus,

$$
P(\text { error }) \geq 1-\frac{2 \pi R \cdot p_{k}}{2 \pi R \cdot p_{k}+l-1}=\frac{l-1}{2 \pi R \cdot p_{k}+l-1} .
$$

From the above proposition, it can be said that the association error is small when the number of previously detected obstacles is small and $p_{k} \gg p_{i}$ for $i \in \mathcal{D}$ and $i \neq k$ in the case when $p_{k}>\frac{1}{2 \pi R}$.

Let us consider the case when $p_{k}<\frac{1}{2 \pi R}$. In this case, the currently detected obstacle is not considered to be one of the previously detected obstacles and is associated with any one of obstacles in the set $\mathcal{D}^{c}$. The probability that the measured data will be associated with wrong obstacle can be expressed as in the next proposition.

Proposition 2 If $p_{k}<\frac{1}{2 \pi R}$, then

$$
P(\text { error })=\frac{2 \pi R \sum_{i \in \mathcal{D}} p_{i}}{2 \pi R \sum_{i \in \mathcal{D}} p_{i}+(l-m)}
$$

where $m$ is the number of previously detected obstacles. 


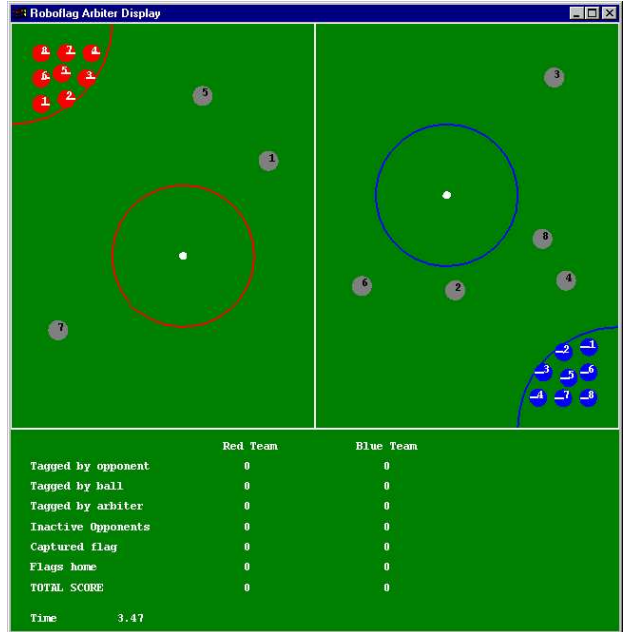

Figure 2: Simulation Platform

Proof: From the Eq. (8), the probability that the currently detected obstacle is not a new one can be expressed as $1-\left(\sum_{j \in \mathcal{D}^{c}} p_{j}\right) /\left(\sum_{j=1}^{l} p_{j}\right)$. This is the probability that the currently detected obstacle will be associated with a wrong obstacle since it is associated with an obstacle in the set $\mathcal{D}^{c}$. Therefore, we have

$$
\begin{aligned}
P(\text { error }) & =1-\frac{(l-m) /(2 \pi R)}{\sum_{i \in \mathcal{D}} p_{i}+(l-m) /(2 \pi R)} \\
& =1-\frac{l-m}{2 \pi R \sum_{i \in \mathcal{D}} p_{i}+(l-m)}
\end{aligned}
$$

From the above proposition, the association error is small when $\sum_{i \in \mathcal{D}} p_{i}$ is small.

In some cases, the sensors on the vehicle might return wrong detection signals. One solution to a such case is to defer the association until the vehicle can recognize they are wrong signals since the probability for consecutive error is smaller than the probability for one time error. We do not consider the case when the sensors on the vehicle return wrong detections constantly.

\section{Simulation}

\subsection{Description of Simulation Platform}

The game named "RoboFlag" was used as a simulation platform for validating the algorithm ([2], [5]). The game has two teams, Red and Blue. The Red team's objective is to infiltrate Blue's territory, grab the Blue flag and bring it back to the Red Home Zone, and visa versa. See Figure 2. During the game, the following objects will be on the playing field: 8 Red robots, 8 Blue robots, 8 scoring balls, and 8 obstacles. Each robot has its own local information such as its position, orientation, translational velocity and angular velocity. It also has information on the position and orientation of obstacles within its conic sensor range with radius $R_{s}$ and angle $\Omega_{r}$. The origin is located at the center of the region and orientation is measured counterclockwise from the horizontal axis with range between 0 and $2 \pi$.

In order to apply the algorithm described in the previous section, two probability density functions, $f\left(m_{T} \mid \mathbf{x}_{T}^{i}\right)$ and $f\left(\mathbf{x}_{T}^{i} \mid \mathbf{x}_{T-1}^{i}\right)$, are needed. The former is the pdf implying that the vehicle will have measurement $m_{T}$ when the obstacle $i$ has the state $\mathbf{x}_{T}^{i}$ at time $T \Delta t$. This is a characteristic of the sensors; thus it can be obtained. The latter is the pdf meaning that the obstacle $i$ will have the state $\mathbf{x}_{T}^{i}$ at time $T \Delta t$ when the obstacle $i$ has the state $\mathbf{x}_{T-1}^{i}$ at time $(T-1) \Delta t$. This can be obtained if there is information on the velocity of obstacles, statistics of angle changes, and so on.

The dynamics of the obstacles are as follows: the initial positions of $l$ moving obstacles are uniformly distributed on the rectangular region $\mathcal{R}$ with (field width $) \times$ (field length). A new random location for each obstacle is determined at time $t_{\text {switch }}$, where $t_{\text {switch }}$ is a Poisson process with parameter $\lambda \Delta t$. Each obstacle moves toward its new target positions with velocity $v_{m}$ at time $t_{\text {switch }}$. The target destination of each obstacle has uniform distribution as well.

\subsection{Simulation Results}

In the simulation, the following values were used: $\Delta t=$ $0.5, \lambda=1.6$, field length $=60$, field width $=40$, and $v_{m}=5$. The region $\mathcal{R}$ is divided into $1 \times 1$ square cells. The vehicle begins with the initial probability map with all cells having values $1 / 4800 \pi$ at time 0 .

Simulation results are shown in Figure 3. The left figure shows the case when the vehicle previously detected one obstacle. The vehicle detected an obstacle (obstacle 1) and the probability distribution of the obstacle's location was estimated by time propagation after the vehicle lost tracking of the obstacle from its sensor range (see the probability distribution on the left of the figure (a)). During navigation through the environment, the vehicle detects an obstacle within its sensor range (see the point indicated by arrow in figure (a)). It recognizes that the obstacle is different from the previously detected one since $p_{1}<1 / 4800 \pi$. The obstacle 2 is added to the set $\mathcal{D}$. From the Eq. (11), the probability of association error is almost zero since $p_{1} \approx 0$, which means the detected obstacle indicated by white arrow is not the previously detected obstacle but a new one.

The right figure shows the case when the vehicle previ- 


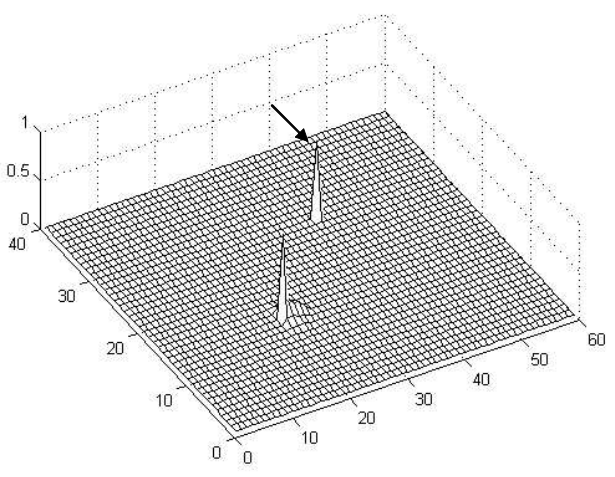

(a)

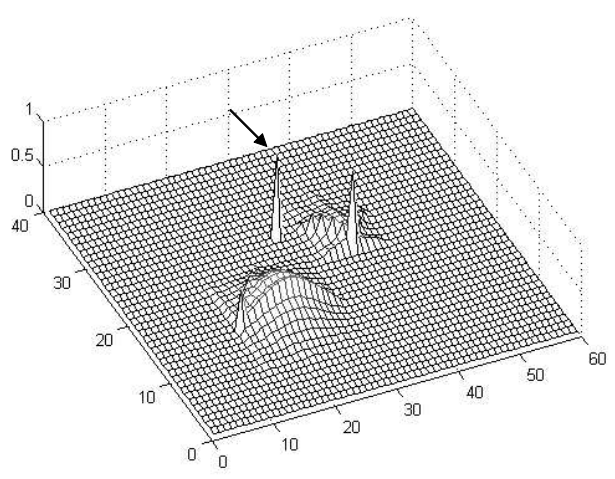

(b)

Figure 3: Simulation Results

ously detected two obstacles, that is, $\mathcal{D}=\{1,2\}$. The probability distribution was updated by time propagation after the vehicle lost tracking of them. The distribution on the left is for obstacle 1 and the one on the right is for obstacle 2 . The vehicle detects an obstacle within its sensor range (see the point indicated by white arrow in figure (b)). It recognizes that the obstacle is different from the previously detected one since $p_{1}<p_{2}<1 / 4800 \pi$.

\section{Conclusion}

This paper proposed a method for building a probability map of uncertain dynamic environments with multiple obstacles. It is assumed that the vehicle has a limited sensor range and therefore lacks global information. The probability map is updated using sensor information and a priori statistics of the dynamic environment. This paper also proposed data association algorithm for indistinguishable obstacles, which enables the vehicle to determine if the detected obstacle at present time is previously detected one or not. This paper also presented error bound of the algorithm.

\section{Acknowledgement}

The authors would like to thank Atif I. Chaudhry for his comments on the manuscript and discussion.

\section{References}

[1] I. J. Cox and J. J. Leonard. Modeling a dynamic environment using Bayesian multiple hypothesis approach. Artificial Intelligence, 66:311-344, 1994.

[2] R. D'Andrea. RoboFlag: Rules and regulation. http://www . mae. cornell . edu/Raff/Research/ Autonomous/RoboFlag/RFrules.pdf, 2002.

[3] A. Elfes. Sonar-based real-world mapping and navigation. IEEE Trans. on Robotics and Automation, RA-3(3):249-265, June 1987.

[4] C. Hue, J.-P. Le Cadre, and P. Pérez. Sequential Monte Carlo methods for multiple target tracking and data fusion. IEEE Trans. on Signal Processing, 50(3):309-325, February 2002.

[5] M. Jun, A. Chaudhry, and R. D'Andrea. The navigation of autonomous vehicles in uncertain dynamic environments: A case study. In Proc. of IEEE Conf. on Decision and Control, Las Vegas, NV, December 2002.

[6] J C. Latombe. Robot Motion Planning. Kluwer Academic Press, 1990.

[7] H. P. Moravec. Sensor fusion in certainty grids for mobile robots. AI Magazine, pages $61-74$, Summer 1988.

[8] D. Schulz, W. Burgard, D. Fox, and A. B. Cremers. Tracking multiple moving objects with a mobile robot. In Proc. of the IEEE Computer Society Conference on Computer Vision and Pattern Recognition (CVPR), 2001.

[9] S. Thrun. Learning metric-topological maps for indoor mobile robot navigation. Artificial Intelligence, 99:21-71, February 1998.

[10] B. Yamauchi and P. Langley. Place recognition in dynamic environments. J. of Robotic Systems, 14:107120, 1997. 